\title{
Cuing effects of faces are dependent on handedness and visual field
}

\author{
Emma Ferneyhough, Damian A. Stanley, \\ Elizabeth A. Phelps, and Marisa Carrasco \\ New York University, New York, New York
}

\begin{abstract}
Faces are unlike other visual objects we encounter, in that they alert us to potentially relevant social information. Both face processing and spatial attention are dominant in the right hemisphere of the human brain, with a stronger lateralization in right- than in left-handers. Here, we demonstrate behavioral evidence for an effect of handedness on performance in tasks using faces to direct attention. Nonpredictive, peripheral cues (faces or dots) directed exogenous attention to contrast-varying stimuli (Gabor patches) - a tilted target, a vertical distractor, or both; observers made orientation discriminations on the target stimuli. Whereas cuing with dots increased contrast sensitivity in both groups, cuing with faces increased contrast sensitivity in right- but not in left-handers, for whom opposite hemifield effects resulted in no net increase. Our results reveal that attention modulation by face cues critically depends on handedness and visual hemifield. These previously unreported interactions suggest that such lateralized systems may be functionally connected.
\end{abstract}

Faces are special visual objects that we encounter every day. Not only are they complex and ever-changing, they are a portal into the thoughts and intentions of others, providing information necessary for navigating our dynamic social world. Perhaps for these reasons, we are particularly responsive to faces; we rapidly evaluate them (Haxby, Hoffman, \& Gobbini, 2002) and use them to make predictions of social outcomes (Oosterhof \& Todorov, 2008). Furthermore, a face has the ability to automatically draw our attention, more so when its expression is fearful rather than neutral (Phelps, Ling, \& Carrasco, 2006). This ability is particularly important, because it is one of the first steps necessary to begin the process of evaluation and prediction formation in our chaotic visual world.

Selective attention can be deployed covertly (without eye movements) to a region in space and improve performance on visual discrimination tasks in that location (Carrasco, 2006; Kinchla, 1992). This is true whether attention is deployed voluntarily (endogenously) or driven involuntarily by a transient change in the visual field (exogenously; see Nakayama \& Mackeben, 1989). Typically, psychophysicists use peripheral cues consisting of dots or bars to direct exogenous attention. When cued with dots or bars, not only does exogenous attention improve performance at cued locations (Carrasco, Penpeci-Talgar, \& Eckstein, 2000; Ling \& Carrasco, 2006), it also impairs performance at uncued locations. This is true even though the cues are uninformative (i.e., they do not predict the target location), and observers are explicitly told that this is the case (Montagna, Pestilli, \& Carrasco, 2009; Pestilli \& Carrasco, 2005). These trade-offs in performance have been interpreted as resulting from the allocation of limited resources. Faces are effective as exogenous cues and can reflexively draw attention to task-relevant locations, perhaps because of their ecological validity and social value (Phelps et al., 2006). However, it is unknown whether there is a corresponding cost, as with dot cues, at irrelevant locations. How do face cues modulate the benefits and costs of attention, at attended and unattended locations, respectively?

On the basis of the finding that face cues confer greater attentional benefit when they depict fearful rather than neutral expressions (Phelps et al., 2006), in a pilot experiment we tested whether we would find both differential benefits (at cued locations) and costs (at uncued locations) for fearful and neutral faces. Although we found no effect of emotion (see the Discussion section), we did discover an intriguing pattern of results mediated by handedness: For left-handers, the cuing effect depended on the location of the target in the visual field.

Interestingly, lesion and imaging studies with righthanders have revealed that face perception and visuospatial attention are hemispherically lateralized. Face recognition is a specialized process of the right hemisphere (Luh, Redl, \& Levy, 1994). Consistent with this finding, people are better at recognizing faces in the left visual field (LVF) than in the right visual field (RVF; see Rhodes, 1985). Greater face-related activity in the right than in the left fusiform face area, as assessed by EEG and fMRI, is thought to underlie this LVF advantage (Yovel, Levy, Grabowecky, \& Paller, 2003; Yovel, Tambini, \& Brandman, 2008). Visuospatial attention is also associated 
with greater activity in the right hemisphere (Siman-Tov et al., 2007), with attention benefiting detection (Fecteau, Enns, \& Kingstone, 2000) and discrimination (Evert, McGlinchey-Berroth, Verfaellie, \& Milberg, 2003) tasks more in the LVF than in the RVF. Correspondingly, more severe attention deficits result from lesions to the right than to the left parietal lobe (Mesulam, 1999). As a group, compared with right-handers, left-handers show more intersubject variability in these lateralized brain functions (e.g., Dronkers \& Knight, 1989; Luh et al., 1994).

It is unknown whether brain lateralization differences observed in right- and left-handers could lead them to exhibit different behavior in experiments that tap into the lateralized functions of face processing and covert attention. Hence, in the present study, using an exogenous attention procedure (Pestilli \& Carrasco, 2005), we systematically investigated how the interaction of handedness and attention cued with faces (Experiment 1) or with dots (Experiment 2) affects visual performance.

\section{METHOD}

\section{Experiment 1}

\section{Effects of Faces As Exogenous Cues}

\section{Participants}

Six right-handed ( 2 males, age range $20-34$ years, $M=26.8$ ) and 6 left-handed ( 1 male, age range $24-31$ years, $M=27.5$ ) observers participated. All had normal or corrected-to-normal vision and completed the 10-item Edinburgh Handedness Inventory (Oldfield, 1971). A score of +100 on the inventory indicates complete right-hand dominance, whereas a score of -100 indicates complete left-hand dominance. Right-handers scored $+78(S D=21)$ and lefthanders scored $-83(S D=14)$, on average.

\section{Apparatus}

Stimuli were presented on a 21-in. ViewSonic P220f monitor $(1,600 \times 1,200$ pixels; $75 \mathrm{~Hz})$ connected to a Power Macintosh
G4 computer via an attenuator. Background luminance was set to $16.5 \mathrm{~cd} / \mathrm{m}^{2}$. During the experiment, each participant's head was stabilized on a chinrest $57 \mathrm{~cm}$ from the monitor.

\section{Stimuli}

Face stimuli consisted of 22 contrast- and luminance-equated grayscale pictures of fearful and neutral faces from the Pictures of Facial Affect series (Ekman \& Friesen, 1976). Gabor patches (sinusoidal gratings in a Gaussian envelope, $S D=1^{\circ} ; 4 \mathrm{cpd}$ ) were created using MATLAB 5.2.1 and the Psychophysics Toolbox (Brainard, 1997). The face cues subtended $4^{\circ} \times 5.3^{\circ}$, and were centered $5^{\circ}$ horizontally and $2.65^{\circ}$ above fixation. The Gabor patches subtended $6^{\circ} \times 6^{\circ}$ and were centered $5^{\circ}$ horizontally and $4^{\circ}$ below fixation. Gabor patch contrast ranged from $3.4 \%$ to $56.7 \%$ in seven log steps. Gabor tilt ranged from $3^{\circ}$ to $6^{\circ}$, chosen for each observer individually based on a $\sim 62.5 \%$-correct criterion in pretesting.

\section{Procedure}

Observers were seated in a darkened room. On each trial, they fixated a central cross for $500 \mathrm{msec}$; then a face cue was presented to the left, right, or on both sides above fixation for $80 \mathrm{msec}$ to manipulate exogenous attention. Following a 53-msec interstimulus interval, one tilted (the target) and one vertical Gabor patch were presented, one on either side below fixation, for $40 \mathrm{msec}$. Participants indicated the target location (left or right) and orientation (counterclockwise or clockwise) with a single buttonpress (Figure 1). Feedback was given after each trial by a high tone for correct and a low tone for incorrect responses. Cues appeared on the same side as targets (valid), the opposite side (invalid), and on both sides (distributed) with equal probability (1/3). Observers completed 3,340 trials on average.

\section{Analysis}

For each condition, we calculated percent correct as a function of contrast. Psychometric functions were fitted using psignifit 2.5.6 (Weibull; http://bootstrap-software.org/psignifit/; Wichmann \& Hill, 2001). Contrast threshold was indexed by the stimulus intensity at which observers were correct $67 \%$ of the time, about halfway between chance $(25 \%)$ and perfect performance $(100 \%)$. The primary dependent variable was contrast sensitivity (CS), which is inverse contrast threshold. Observers' CS scores were individually normalized by dividing each condition mean by the average of all conditions
Experiment 1

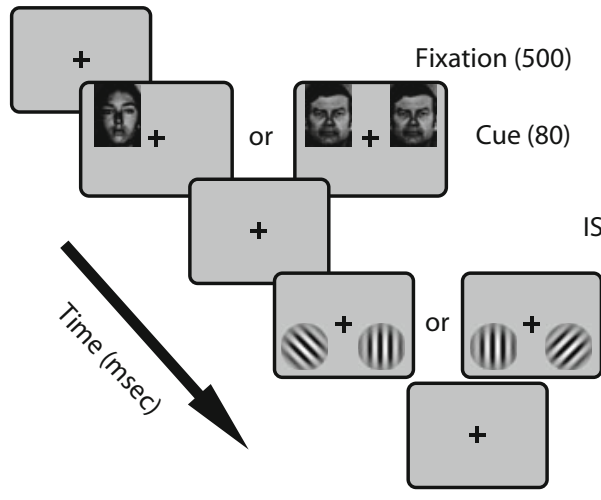

"Report location and orientation of tilted target Gabor patch"
Experiment 2
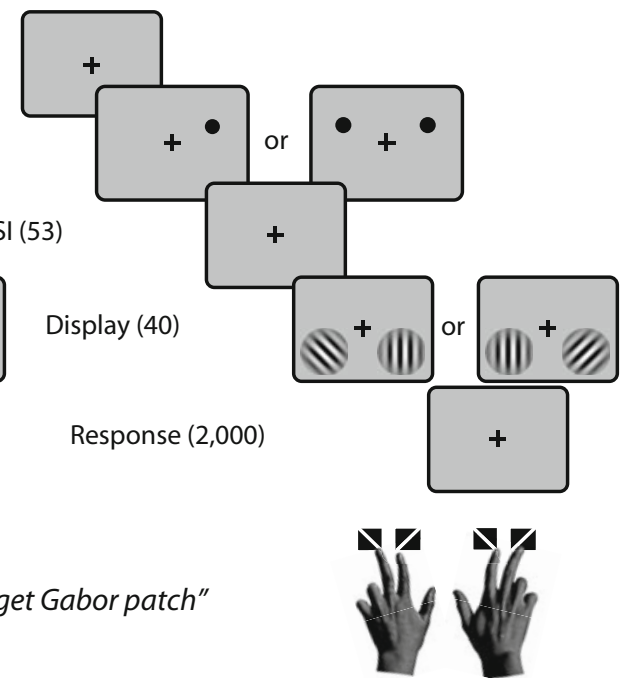

Figure 1. Trial sequence for Experiment 1 (face cues) and Experiment 2 (dot cues). Images not to scale; contrast and target Gabor tilt emphasized for clarity. 
to reduce the influence of baseline CS differences across observers. Normalized CS scores were then averaged across observers in each handedness group. Reaction times (RTs) were also measured.

\section{Experiment 2}

\section{Effects of Dots As Exogenous Cues (Control)}

All experimental parameters for Experiment 2 were the same as for Experiment 1, except for the following: (1) 5 out of 6 observers from each handedness group in Experiment 1 participated in Experiment $2 ;(2)$ black dot cues $\left(0.3^{\circ}\right.$ diameter, $1^{\circ}$ above and $5^{\circ}$ horizontally from fixation) were used instead of face cues; (3) Gabor targets were always tilted $\pm 4^{\circ}$; and (4) observers completed 4,000 trials on average.

\section{RESULTS}

Given that there were no differences in performance or RT between facial expression conditions (fearful vs. neutral face cues, $p>.1$ ), the data were averaged across both expressions. Here we report detailed statistics for CS, and note that the RT analyses showed no speed-accuracy trade-offs for any comparison. For each experiment there are two within-subjects factors: cue validity (valid, distributed, invalid) and visual field (LVF, RVF). There is also one between-subjects factor: handedness (left, right).

To determine whether cue validity interacted with handedness and visual field and whether this interaction depended on cue type, three-way mixed factorial ANOVAs were performed for face and dot data separately, with cue validity, visual field, and handedness as factors. There was a significant interaction of the three factors for faces $[F(2,20)=14.349, p<.001]$ but not for dots $[F(2,16)=$ $1.652, p>.10]$. To better understand how faces are different from dots, we first presented an analysis of the effect of cue type alone. Then, because our pilot study indicated that left-hander performance depended on the target location in the visual field, we investigated the effect of handedness, as well as the effect of visual field. Lastly, we examined the relation between degree of handedness and cue validity effect.

\section{Effects of Cue Type}

To evaluate the effect of cue type, CS was averaged over both visual fields and handedness conditions separately for face and dot cue data (Figure 2A). One-way repeated measures ANOVAs performed on cue validity (valid, distributed, or invalid) indicated that it changed CS marginally when faces were used $[F(2,22)=3.091, p=.066$, $\left.\eta_{\mathrm{RM}}^{2}=.22\right]$; however, it changed CS significantly when dots were used $\left[F(2,18)=10.663, p<.001, \eta_{\mathrm{RM}}^{2}=.54\right]$. The results replicated previous findings for dots (Carrasco et al., 2000; Ling \& Carrasco, 2006; Pestilli \& Carrasco, 2005), but not for faces (Phelps et al., 2006). Faces decrease the magnitude of the cue validity effect but, at this point in the analysis, it is unclear why this is the case.

\section{Effects of Handedness}

We then split the data to evaluate the effect of handedness. When right-handers were cued with either faces or dots, cue validity significantly changed CS [face,

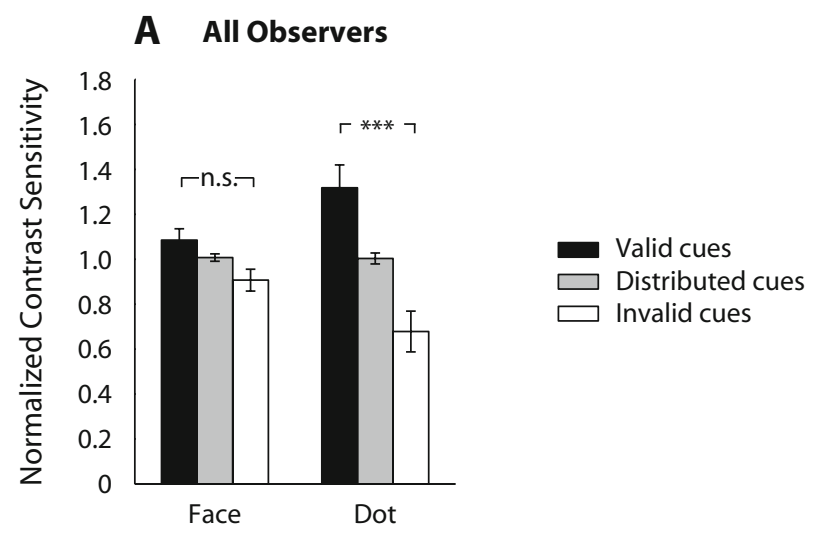

B Left-Handers

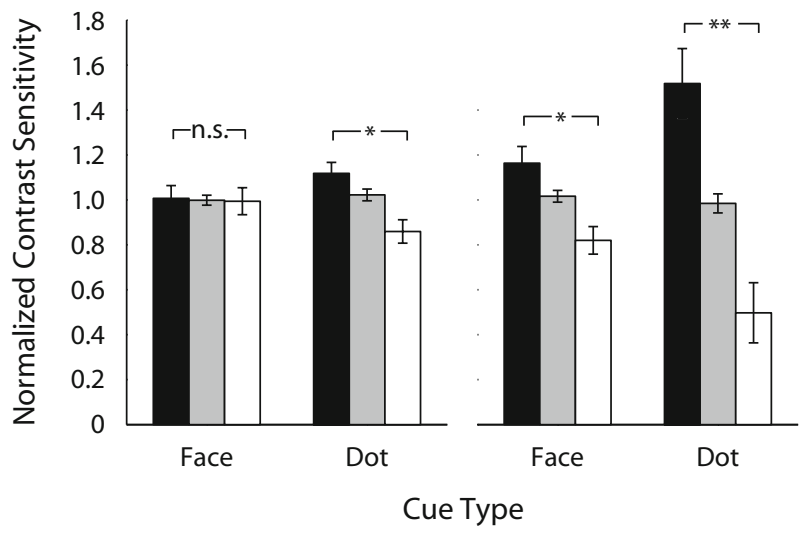

Figure 2. (A) Contrast sensitivity data for all observers (face $n=12$; dot $n=10$ ), averaged over handedness group and target visual field. (B) Contrast sensitivity data split by handedness group (each group: face $n=6 ; \operatorname{dot} n=5$ ). Error bars are \pm standard errors of the means. ${ }^{*} p<.05 .{ }^{* *} p<.01 .{ }^{* * * *} p<.001$.

$F(2,10)=5.992, p<.02, \eta_{\mathrm{RM}}^{2}=.55 ; \operatorname{dot}, F(2,8)=$ $11.838, p<.005, \eta_{\mathrm{RM}}^{2}=.75$ ] (Figure 2B). Although when left-handers were cued with dots cue validity significantly changed $\operatorname{CS}\left[F(2,8)=5.904, p<.05, \eta_{\mathrm{RM}}^{2}=.6\right]$, this was not the case when they were cued with faces $[F(2,10)<1]$. The decreased magnitude of the initial face-cue validity effect across handedness appears to be due to the lack of CS modulation in left-handers when cued with faces.

\section{Effects of Handedness and Visual Field}

Next, to evaluate the effect of visual field, the data were split on the basis of whether the target appeared on the left or right side of the screen. Within each handedness group, two-way repeated measures ANOVAs were performed on target visual field (LVF, RVF) and cue validity (valid, distributed, invalid) separately for face and dot cues. Left-hander face data revealed a significant interaction between VF and cue validity $[F(2,10)=6.519, p<$ $.02]$ (Figure 3A). When targets were in the LVF, valid face cues resulted in the highest CS, followed by distributed and invalid cues ( $M=1.15,1.10$, and 1.01, respectively). However, a different pattern was found when targets were 

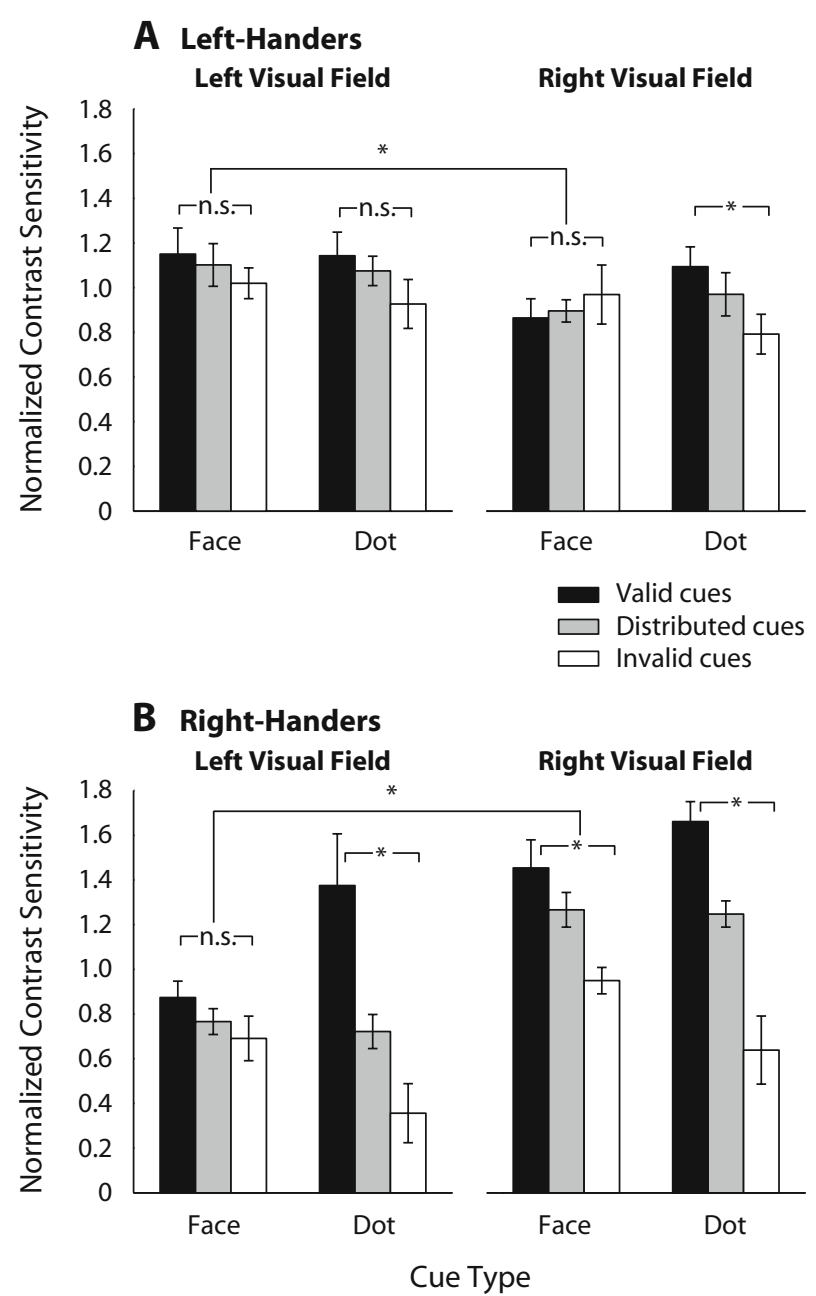

Figure 3. Contrast sensitivity data from Figure 2B, split by target visual field. (A) Left-hander face $(n=6)$ and $\operatorname{dot}(n=5)$ data. (B) Right-hander face $(n=6)$ and $\operatorname{dot}(n=5)$ data. Error bars are \pm standard errors of the means. ${ }^{*} p<.05$.

in the RVF: Invalid cues led to the highest CS, followed by distributed and valid cues $(M=0.97,0.90$, and 0.86 , respectively). In contrast, left-hander dot data revealed a main effect of cue validity $[F(2,8)=5.905, p<.05]$ with valid dot cues leading to the highest $\mathrm{CS}$, followed by distributed and invalid cues $(M=1.12,1.02$, and 0.86 , respectively). VF and cue validity did not significantly interact $[F(2,8)=1.699, p>.10]$.

Right-hander face data revealed a significant interaction of VF and cue validity $[F(2,10)=7.93, p<.01]$ (Figure 3B). CS was higher in the RVF than in the LVF, and there were greater differences in CS due to cue validity in the RVF than in the LVF (valid - invalid CS $=0.5$ and 0.18 , respectively). Right-hander dot data revealed a main effect of VF $[F(2,8)=18.954, p<.02]$, with CS being higher in the RVF than in the LVF $(M=1.18$ and 0.82 , respectively). There was also a main effect of cue validity $[F(2,8)=11.837, p<.005]$, with valid cues resulting in the highest CS, followed by distributed and invalid cues ( $M=1.52,0.98$, and 0.5 , respectively). VF and cue validity did not significantly interact $[F(2,8)=$ $1.5, p>.10]$.

Lastly, we examined the correlation between each individual's cue validity effect (valid - invalid CS) and their handedness score (Figure 4). These two indices were positively and significantly correlated when targets appeared in the RVF with both face $\left(R^{2}=.56, p<.01\right)$ and $\operatorname{dot}\left(R^{2}=.45, p<.05\right)$ cues; however, it appears that these correlations may each be due to different underlying mechanisms (see the Discussion section). When targets appeared in the LVF, this effect was marginal with dot cues $\left(R^{2}=.38, p=.058\right)$, but no such correlation emerged with face cues $\left(R^{2}=.03, p>.1\right)$.

\section{DISCUSSION}

Does covert attention evoked by face and dot cues have comparable benefits and costs on contrast sensitivity? Critically, the answer to this question depends on the observer's handedness. For right-handers, both faces and dots are effective at eliciting attention, resulting in a benefit at cued locations and a cost at uncued locations (see Figure 2B, third and fourth triplet from L to R). Conversely, for left-handers, faces and dots elicit attention differentially: Whereas dot cues result in enhanced CS with attention, face cues have a different pattern in each hemifield. When faces cued LVF targets, valid cues increased $\mathrm{CS}$, and invalid cues decreased CS, relative to distributed cues. However, when faces cued RVF targets, valid cues decreased $\mathrm{CS}$ and invalid cues increased $\mathrm{CS}$ relative to distributed cues (see Figure 3A, first and third triplet from $\mathrm{L}$ to $\mathrm{R}$ ). Consequently, averaging over both hemifields resulted in no net effect of cue validity in left-handers (see Figure 2B, first triplet on left).

Previous studies have shown that when exogenous attention is manipulated via dot or bar cues, it elicits attentional benefits and costs at cued and uncued locations, respectively (Carrasco et al., 2000; Montagna et al., 2009; Pestilli \& Carrasco, 2005), and that face cues elicit attentional benefits at cued locations (Phelps et al., 2006). The present study replicated previous dot cue findings for all observers, and revealed that, for right-handers, the benefits of face cues were accompanied by costs at the uncued locations. These findings support selective attention's role in helping to manage limited resources that result in processing trade-offs (Carrasco, 2006; Kinchla, 1992; Pestilli \& Carrasco, 2005).

Although we had expected both benefits and costs of attention on CS to be mediated by facial expression (Phelps et al., 2006), no such differences emerged. A recent study suggests a possible explanation: The valence effect of facial expression interacts with Gabor spatial frequency. There is no advantage of fearful faces on the perception of oriented stimuli with spatial frequency greater than $2 \mathrm{cpd}$ (Bocanegra \& Zeelenberg, 2009). These results suggest that the beneficial effects of emotion are restricted to low spatial frequencies. Whereas in our previous study we used 2-cpd stimuli, in the present 


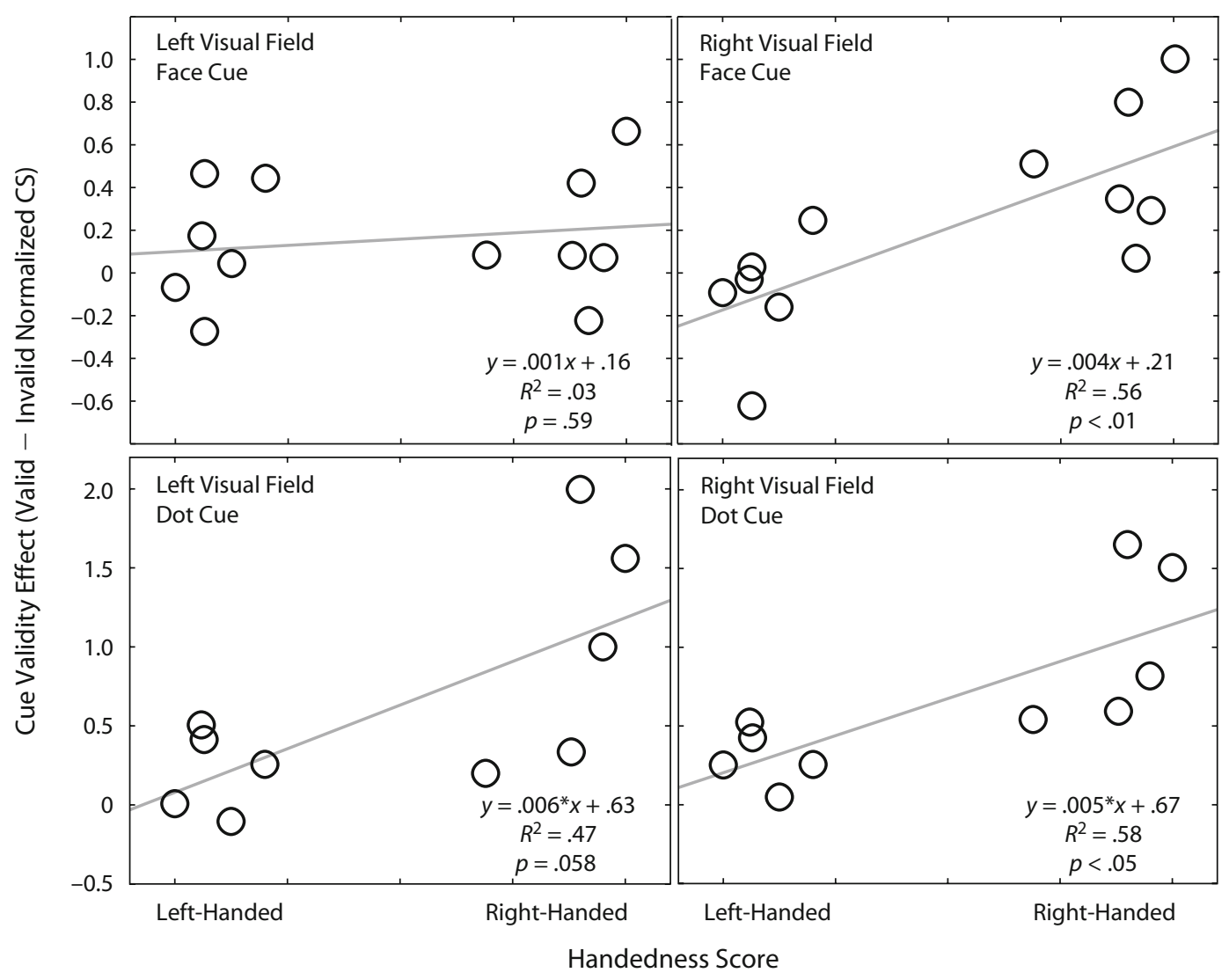

Figure 4. Correlation of handedness score with cue validity effect. For RVF targets cued with faces, this significant correlation is driven by both a decrease in attention effect in left-handers and an increase in attention effect in right-handers, whereas for dots, the significant correlation is driven mostly by closer clustering of attention effect in right-handers.

experiment we used 4-cpd stimuli, which may have resulted in the null effect.

Could cue complexity, rather than "faceness," account for the visual field and handedness effects? A recent study suggests that the effect is face-specific. Face cues produce greater differences in RT to detect cued and uncued targets than equivalently complex phase-scrambled and inverted faces, but only in the RVF (Elder, Balaban, Kamyab, Wilcox, \& Hou, 2008). Consistent with that study, the present results show that performance with face cues is also affected by visual field asymmetries. One aspect of the data that can be explained by differential cue complexity is that, in general, dot cues result in overall greater cue validity effects and contrast sensitivity than do face cues. To make expressions discriminable, the faces are much larger than the dots, which may have resulted in a more diffuse attention boost due to the trade-off between attention field size and spatial resolution (Eriksen \& St. James, 1986). However, the contrast and luminance of the faces were equated across the whole set, resulting in much lower contrast for faces than for dots, which could also make them less effective exogenous cues (Fuller, Park, \& Carrasco, 2009).

Furthermore, the present results show that the cue validity effects are stronger in the RVF for right-handers and in the LVF for left-handers. This is consistent with the dominant-hand attentional bias seen in the Simon effect (Rubichi \& Nicoletti, 2006), which reflects an interaction of target location and the location of the hand used to make the response on RT. Responses are faster when made with the hand adjacent to the target, rather than with the opposite hand. A larger Simon effect is observed in the hemifield corresponding to the dominant hand: For right-handers, the difference in RT between hands to make an RVF response is larger than the corresponding difference for an LVF response. Crucially, right-handers have faster RT for RVF targets when they respond with their right hands and slower RT for LVF targets when they respond with their left hands (vice versa for left-handers). This effect is thought to be due to spatial attention, which allows a more efficient response selection for the dominant hand. In the present experiments, this attentional bias may also explain the increased CS and cue validity effects in the visual fields corresponding to each group's dominant hand.

The degree of handedness and the magnitude of the attention effect were significantly correlated in the RVF for both cue types, whereas the LVF correlation was insignificant for faces and only marginal for dots (Figure 4). However, the significant RVF correlations for faces versus dots 
may have different underlying phenomena. The significant face correlation is driven by both a decrease in lefthanders' and an increase in right-handers' cue validity effect (Figure 4, top-right panel); the use of face cues seems to affect attentional deployment to the RVF in opposing ways in these two groups. This pattern of results is consistent with the difference in degree of lateralization and intersubject variability for these two groups (Boles, 1989; Luh et al., 1994), especially with regard to face processing (Bourne, 2008). In contrast, the significant dot correlation is primarily driven by closer clustering of right-handers' cue validity effect, with no real change in left-handers' cue validity (Figure 4, bottom-right panel); this finding indicates that attentional deployment to the LVF results in an increase of CS for everyone, but to different degrees. This pattern of results is consistent with the existence of attentional asymmetries across the visual field (e.g., Fecteau et al., 2000), which depends in part on handedness (i.e., the Simon effect; Rubichi \& Nicoletti, 2006).

Regardless of the differences between visual fields, why might the effect of faces on covert exogenous attention depend on handedness? It is possible that in left-handers' brains, attention-related signals have to travel farther to boost the processing of spatially specific locations cued by faces than those cued by dots. The right hemisphere of the right-hander's brain is dominant for both face and attention processing, allowing for efficient interactions of face cues and attention signals. However, given their variability in degree of lateralization, the functions of lefthanded brains may be more distributed, leading to greater distances between face- and attention-related regions. As a result, left-handers as a group may not experience the same benefits and costs of attention on CS, when cued with faces, as right-handers.

Even though left-handers comprise $10 \%$ of the population (Raymond, Pontier, Dufour, \& Møller, 1996), they are excluded from most cognitive psychology and cognitive neuroscience studies, because researchers are concerned with laterality issues. Conversely, visual perception data from right- and left-handers are usually averaged. However, we show here that handedness is a critical variable affecting not only higher cognitive processes but also perception. Our visual systems have evolved to become "face recognition experts," a specialization that interacts differently with attention in right- and left-handers.

\section{AUTHOR NOTE}

We thank Sam Ling and David Carmel, as well as other Carrasco Lab members, for their helpful comments. This research was funded by Grants NIH R01-EY016200 to M.C. and NIH R01-MH062104 to E.A.P. Correspondence concerning this article should be addressed to E. Ferneyhough, Psychology Department, New York University, 6 Washington Place, New York, NY 10003 (e-mail: emmafern@nyu.edu).

\section{REFERENCES}

Bocanegra, B. R., \& Zeelenberg, R. (2009). Emotion improves and impairs early vision. Psychological Science, 20, 707-713. doi:10 $.1111 / \mathrm{j} .1467-9280.2009 .02354 . x$

BoLEs, D. B. (1989). Do visual field asymmetries intercorrelate? Neuropsychologia , 27, 697-704. doi:10.1016/0028-3932(89)90114-0

Bourne, V. J. (2008). Examining the relationship between degree of handedness and degree of cerebral lateralization for processing facial emotion. Neuropsychologia, 22, 350-356. doi:10.1037/0894 $-4105.22 .3 .350$

Brainard, D. H. (1997). The Psychophysics Toolbox. Spatial Vision, 10, 433-436. doi:10.1163/156856897X00357

CARrasco, M. (2006). Covert attention increases contrast sensitivity: Psychophysical, neurophysiological and neuroimaging studies. Progress in Brain Research, 154, 33-70.

Carrasco, M., PenPeci-Talgar, C., \& Eckstein, M. P. (2000). Spatial covert attention increases contrast sensitivity across the CSF: Support for signal enhancement. Vision Research, 40, 1203-1215. doi:10.1016/ S0042-6989(00)00024-9

Dronkers, N. F., \& KNight, R. T. (1989). Right-sided neglect in a left-hander: Evidence for reversed hemispheric specialization of attention capacity. Neuropsychologia, 27, 729-735. doi:10.1016/0028 $-3932(89) 90118-8$

Ekman, P., \& Friesen, W. (1976). Pictures of facial affect. Palo Alto, CA: Consulting Psychologists Press.

Elder, J. H., Balaban, D. Y., Kamyab, A., Wilcox, L., \& Hou, Y. (2008). Selectivity for faces as exogenous attentional cues [Abstract]. Journal of Vision, 8(6), 685a.

Eriksen, C. W., \& St. JAMES, J. D. (1986). Visual attention within and around the field of focal attention: A zoom lens model. Perception \& Psychophysics, 40, 225-240.

Evert, D. L., McGlinchey-Berroth, R., Verfaellie, M., \& MilBERG, W. P. (2003). Hemispheric asymmetries for selective attention apparent only with increased task demands in healthy participants. Brain \& Cognition, 53, 34-41. doi:10.1016/S0278-2626(03)00207-0

Fecteau, J. H., Enns, J. T., \& Kingstone, A. (2000). Competitioninduced visual field differences in search. Psychological Science, 11, 386-393. doi:10.1111/1467-9280.00275

Fuller, S., Park, Y., \& Carrasco, M. (2009). Cue contrast modulates the effects of exogenous attention on appearance. Vision Research, 49, 1825-1837. doi:10.1016/j.visres.2009.04.019

HaXby, J. V., Hoffman, E. A., \& Gobbini, M. I. (2002). Human neural systems for face recognition and social communication. Biological Psychiatry, 51, 59-67.

Kinchla, R. A. (1992). Attention. Annual Review of Psychology, 43, 711-742. doi:10.1146/annurev.ps.43.020192.003431

Ling, S., \& CARrasco, M. (2006). Sustained and transient covert attention enhance the signal via different contrast response functions. Vision Research, 46, 1210-1220. doi:10.1038/nn1761

LuH, K. E., RedL, J., \& LEVY, J. (1994). Left- and right-handers see people differently: Free-vision perceptual asymmetries for chimeric stimuli. Brain \& Cognition, 25, 141-160. doi:10.1006/brcg.1994.1028

Mesulam, M. M. (1999). Spatial attention and neglect: Parietal, frontal and cingulate contributions to the mental representation and attentional targeting of salient extrapersonal events. Philosophical Transactions of the Royal Society B, 354, 1325-1346.

Montagna, B., Pestilli, F., \& Carrasco, M. (2009). Attention trades off spatial acuity. Vision Research, 49, 735-745. doi:10.1016/j visres.2009.02.001

Nakayama, K., \& Mackeben, M. (1989). Sustained and transient components of focal visual attention. Vision Research, 29, 1631-1647. doi:10.1016/0042-6989(89)90144-2

OLDFIELD, R. C. (1971). The assessment and analysis of handedness: The Edinburgh inventory. Neuropsychologia, 9, 97-113. doi:10.1016/0028 -3932(71)90067-4

Oosterhof, N. N., \& Todorov, A. (2008). The functional basis of face evaluation. Proceedings of the National Academy of Sciences, 105, 11087-11092. doi:10.1073/pnas.0805664105

Pestilli, F., \& Carrasco, M. (2005). Attention enhances contrast sensitivity at cued and impairs it at uncued locations. Vision Research, $\mathbf{4 5}$, 1867-1875. doi:10.1016/j.visres.2005.01.019

Phelps, E. A., Ling, S., \& CARrasco, M. (2006). Emotion facilitates perception and potentiates the perceptual benefits of attention. Psychological Science, 17, 292-299. doi:10.1111/j.1467-9280.2006.01701.x

Raymond, M., Pontier, D., Dufour, A., \& Møller, A. P. (1996). Frequency-dependent maintenance of left-handedness in humans. Proceedings of the Royal Society B, 263, 1627-1633.

RHodes, G. (1985). Lateralized processes in face recognition. British Journal of Psychology, 76, 249-271.

Rubichi, S., \& Nicoletti, R. (2006). The Simon effect and handed- 
ness: Evidence for a dominant-hand attentional bias in spatial coding Perception \& Psychophysics, 68, 1059-1069.

Siman-Tov, T., Mendelsohn, A., Schonberg, T., Avidan, G., PodLIPSKY, I., PESSOA, L., ET AL. (2007). Bihemispheric leftward bias in a visuospatial attention-related network. Journal of Neuroscience, 27, 11271-11278. doi:10.1523/JNEUROSCI.0599-07.2007

Wichmann, F. A., \& Hill, N. J. (2001). The psychometric function: I. Fitting, sampling, and goodness of fit. Perception \& Psychophysics, 63, 1293-1313.

Yovel, G., Levy, J., Grabowecky, M., \& Paller, K. A. (2003). Neural correlates of the left-visual-field superiority in face perception appear at multiple stages of face processing. Journal of Cognitive Neuroscience, 15, 462-474. doi:10.1162/089892903321593162

Yovel, G., Tambini, A., \& Brandman, T. (2008). The asymmetry of the fusiform face area is a stable individual characteristic that underlies the left-visual-field superiority for faces. Neuropsychologia, 46, 3061-3068. doi:10.1016/j.neuropsychologia.2008.06.017

(Manuscript received September 19, 2009; revision accepted for publication January 22, 2010.) 